\author{
Chrischta Ganz
}

\title{
Ganzheitliche Ansätze bei chronischen Erkrankungen
}

Bei chronischen Krankheiten versucht das gesamte Betreuungsnetz aus Ärztinnen und Ärzten, Therapeutinnen und Therapeuten sowie dem Pflegepersonal das Kranksein zu erfassen und $\mathrm{zu}$ verstehen - am liebsten mit klar diagnostizierten Erkrankungen. In der hochentwickelten Diagnostik der heutigen Medizin wird jedoch manchmal übersehen, dass die Genauigkeit der Diagnostik auch verhindern kann, dass der Blick auf das eigentliche Kranksein und dessen Bedeutung fällt. Der Mensch als Ganzes kann dabei aus dem Blick fallen. Gerade wenn die ausgefeilten Therapien bei chronischen Erkrankungen nicht anschlagen, sind Heilmittel und Therapien gefragt, die den Menschen und nicht Krankheitsbilder behandeln, damit die Patientin bzw. der Patient die Verbindung zu sich selbst wieder findet.

\section{Chronische Entzündungen}

Als Ursache vieler chronischer Erkrankungen vermutet man systemische entzündliche Prozesse [1]. Hier wird eine ganzheitliche Betrachtungsweise noch essenzieller, um eine adäquate und erfolgreiche Therapie zu finden. Eine Entzündung ist vorerst ein natürlicher immunlogischer Schutzmechanismus des Körpers, der erst aufgrund bestimmter Faktoren zum Problem werden kann. Neben der akuten Entzündung mit den klassischen Entzündungszeichen Rötung, Schwellung, Schmerz, Erwärmung und Funktionseinschränkung gibt es die stille Inflammation, die oft «unbemerkt» verläuft. Zu den stillen Inflam- mationen gehört auch die sogenannte Immunoseneszenz, auch Inflammaging oder Entzündungsaltern genannt, die durch eine Erhöhung des GammaInterferon-Spiegels und eine Verminderung der adaptiven Immunabwehr gekennzeichnet ist [1].

Die Ursachen für vermehrte chronische Entzündungen liegen vermutlich in der höheren Lebenserwartung sowie in den veränderten Lebensbedingungen seit der Industrialisierung. Als Auslöser für stille Entzündungen werden eine Vielzahl an Reizen genannt, die Stress und Gewebeschädigung hervorrufen. Eine wichtige Funktion in dieser Entzündungskaskade liegt bei einem Multi-Proteinkomplex, dem Inflammasaom. Nach Ausschüttung des sogenannten DAMP («danger-associated molecular pattern») bei stillen Entzündungen bzw. des PAMP («pathogen-associated molecular pattern») bei Entzündungen, die durch Pathogene ausgelöst werden, binden diese an spezifische Erkennungsrezeptoren und an zytosolische Proteine: Der Immunkomplex Inflammasom entsteht. In seiner aktivierten Form vermittelt er die Ausschüttung der Zytokine Interleukin1-Beta und Interleukin-18, die den entzündlichen Prozess auslösen. Weiter werden Zytokine wie der Tumornekrosefaktor-alpha und Interferongamma, zelltoxische Sauerstoff- und Stickstoffradikale und stark proteolytische Enzyme aktiviert, sodass schwere Gewebeschäden entstehen, die ihrerseits das Inflammason und den gesamten Entzündungsprozess anregen und stabilisieren [1]. Die Ingangsetzung und das Aufrechterhalten von

\author{
Kasten. Salutogenese [6] \\ Von griech. «salus» für «Gesundheit» und griech. \\ «genese» für «Entstehung». \\ Der Begriff wurde vom israelisch-amerikanischen \\ Medizinsoziologen Aaron Antonovsky (1923-1994) \\ in den 1970er-Jahren als Gegenbegriff zur Pathoge- \\ nese formuliert und in die Wissenschaft einge- \\ bracht. \\ Bei der Salutogenese stehen die Ressourcen des ein- \\ zelnen Menschen im Vordergrund. Salutogenese ist \\ somit als «Schatzsuche» und nicht etwa als «Fehler- \\ fahndung» $\mathrm{zu}$ verstehen. \\ Die Salutogenese behandelt Themen wie: \\ - Warum werden einige Menschen krank, andere \\ nicht? \\ - Was stärkt die Gesundheit und die individuellen \\ Selbstheilungskräfte? \\ - Wie geht der Mensch mit seiner Gesundheit um? \\ - Fragen zur Lebensbewältigung. \\ - Umgang mit existenziellen Lebensfragen.
}

Entzündungen unterliegen somit äusserst komplexen biochemischen Immunprozessen.

Die wichtigste Therapie besteht darin, die Entzündungsreaktion durch das Eliminieren der Trigger anzuhalten. Die Suche nach den entsprechenden «triggernden» Faktoren kann sich als schwierig und äusserst aufwendig herausstellen. Um den Entzündungsprozess zu stoppen, sollen gezielte naturheilkundliche Therapien zur Unterstützung des Immungleichgewichts angewendet werden.

\section{Autoimmunerkrankungen}

Mit chronischen Erkrankungen gehen Autoimmunerkrankungen einher. Im Organismus zirkulierende Immunkomplexe führen zur Freisetzung von Komplementen bzw. chemischen Mediatoren und von freien Radikalen.

\section{KARGER}

(c) 2015 S. Karger GmbH, Freiburg

Fax +497614520714
Chrischta Ganz

Praxis für Naturheilkunde

Friedhofstrasse 15, 8636 Wald ZH, Schweiz

naturheilpraxis@chrischtaganz.ch

www.chrischtaganz.ch 
Damit verbundene Folgen sind chronische Entzündungen, Gewebezerstörung, Schmerzen, Mobilitätsverluste, Funktionsstörungen und Knochendemineralisierung.

Grundprinzip der schulmedizinischen Behandlung ist die Unterdrückung der körpereigenen Abwehr mittels Cortison, nichtsteroidalen Antirheumatika, Immunsuppressiva, Matrix-Metalloproteinasen, monoklonalen Antikörpern usw. sowie deren Kombinationsbehandlung.

Einfluss auf die Erkrankungen nehmen Genetik, Alter, immunbelastende Faktoren wie Fehler in Ernährung und Lebensstil, Genuss- und Suchtmittel, Umweltgifte (Schwermetalle, organische Substanzen wie Abgase, Pestizide), Lebensmittelzusatzstoffe, Infektionsherde, Medikamente und andere Noxen [2].

Durch die glykosylierten und oxidativ veränderten Proteine werden das immunologische Gleichgewicht (und damit das Reparatur- und Entsorgungssystem des Körpers, unter anderem das Glutathionsystem) und das Gleichgewicht zwischen freien Radikalen und Antioxidantien (oxidativer Stress) ungünstig verschoben [3]. Damit verbunden ist eine vermehrte Freisetzung von Entzündungsparametern, die Interaktionen im Mikronährstoffhaushalt sowie eine starke Beeinträchtigung des intestinalen Immunsystems (Senkung des sekretorischen IgA und der T-Lymphozyten in der Darmmukosa, Störung der physiologischen Darmflora, erhöhte Darmpermeabilität und Resorptionsstörungen) auslösen [1, 2]. Zucker in allen Formen (Glukose, Fruktose, Sukrose) stört die Leukozyten in ihrer immunologischen Aktivität.

\section{Schmerz}

Schmerz ist einer der häufigsten Gründe, warum sich ein Mensch in ärztliche oder komplementärmedizinische Behandlung begibt. Schmerzmittel gelten als die am häufigsten verkauften Medikamente [4]. Wenn es um
Schmerzbehandlung geht, stehen an erster Stelle folgende Fragen: Was ist Schmerz? Welchen Sinn hat Schmerz? Welche Auswirkungen hat Schmerz auf den menschlichen Organismus?

Schmerz gilt als rein subjektives Gefühl, das ein Individuum wahrnimmt und (meist) als unangenehme Empfindung einstuft. Die Beschreibung des Schmerzes ist somit kein «objektiv messbarer Parameter». Schmerz macht keine Aussage über die tatsächliche Verletzung des Körpers, der Seele oder des Geistes. Allerdings zeigt Schmerz auf, dass der Mensch in seiner Integrität verletzt wird oder verletzt worden ist. Daraus folgt, dass genauso wie das Schmerzempfinden auch jede Schmerztherapie individuell gestaltet werden muss. Eine Schmerztherapie, die bei allen Schmerzpatienten wirkt, gibt es nicht.

Chronischer Schmerz gilt heute als eigenständige Krankheit, wenn die Schmerzen länger als 6 Monate bestehen bzw. länger andauern als die erwartete Heilungszeit. Chronische Schmerzen verändern (neben der verminderten Lebensqualität) die Interpretation der Signale im Hypothalamus und führen oft zu einer erniedrigten Schmerzempfindung, woraufhin sich die Schmerzsymptomatik verschlimmert [5]. Michael Schünemann schreibt dazu: «Die Reizung eines Schmerzrezeptors führt immer zur Irritation umliegender Schmerzrezeptoren, sodass sich der Schmerz in einem grösseren Areal bemerkbar macht. Ausserdem regt die Reizung von Nozizeptoren deren Vermehrung im entsprechenden Gewebe an, wodurch die Schmerzempfindlichkeit steigt. So kann eine erneute Schädigung früher erkannt werden, allerdings wird so einer eventuellen Chronifizierung der Weg gebahnt» [5]. Diesen Teufelskreis gilt es zu durchbrechen.

Schmerz empfindet der Mensch nicht nur als objektive Information, sondern er interpretiert und gestaltet die Information und übersetzt sie in eine subjektive Qualität. Einfach nachvollziehbar ist diese Tatsache bei der unterschiedlichen «Bewertung» von Kopfweh nach einer fröhlich durchzechten Nacht oder nach der Diagnose Hirntumor [6]. Mögliche Erklärungsansätze liegen in der sogenannten Gate-Control-Theorie, die aufzeigt, dass viele Faktoren die Schmerz- und Krankheitswahrnehmung beeinflussen, beispielsweise:

- soziokulturelle Aspekte;

- kognitive Faktoren («Es tut jetzt einen Moment lang weh, dafür wird es nachher wieder gut.»);

- die eigene Wertung der Situation;

- Verdrängungs- und Bewältigungsstrategien;

- Hilflosigkeit, Ohnmacht und das Gefühl von Ausgeliefertsein;

- Schmerz- und Krankheitsfixierung;

- Depression, Angst;

- Stress, Aggression, Anspannung;

- sowie eine negative Lebenseinstellung [6].

Über afferente Nervenfasern weitergeleitet, erreicht die Schmerzinformation den Thalamus, wo je nach Reizzustand und Stimmung die spätere Schmerzempfindung moduliert wird. Hier geschieht somit bereits eine individuelle Interpretation und Wahrnehmung des Schmerzes. Der Thalamus leitet die Information der Schädigung, sei sie endogen oder exogen verursacht, an das limbische System und an die Grosshirnrinde weiter, wo noch einmal eine Anpassung an die psychische Situation und an die Lebenserfahrungen des Individuums geschieht. Hier treten alle mit dem Schmerz in Verbindung stehenden körperlichen Mechanismen in Aktion: Atemtätigkeit, Herzfrequenz, Blutdruck und viele andere mehr. Allein schon unter diesen Gesichtspunkten ergeben Begleittherapien wie Atemtherapie, Yoga, Feldenkrais, Tai Chi, Meditation, autogenes Training oder auch Entspannungsmethoden Sinn.

\section{Therapie}

Mit jeder chronischen Erkrankung werden sowohl die Patientinnen und 
Patienten als auch die Ärzte, Therapeuten und Naturheilpraktiker mit der Sterblichkeit konfrontiert (Abb. 1). Dies kann die Behandlung chronischer Erkrankungen zusätzlich erschweren, eröffnet aber für eine ganzheitliche Therapie auch neue Wege. Der von einer chronischen Erkrankung betroffene Mensch wie auch die behandelnden Medizinfachpersonen sind in ihrer Eigenverantwortung herausgefordert, damit die eigene Gestaltungsmacht ebenso anerkannt wird wie die Grenzen des menschlichen Einflusses auf das Leben [6].

Ressourcen und Selbstheilungskräfte Jede ganzheitliche Therapie möchte die Selbstheilungskräfte anregen und der Patientin bzw. dem Patienten seine Eigenverantwortung zurückgeben. Dabei muss zwingend das abhängige Modell der Beziehung zwischen Patient und Arzt bzw. Therapeut verabschiedet werden (Tab. 1).

Jeder Mensch, auch der chronisch kranke, kennt viele Strategien, um seine Ressourcen und Reorganisationsmechanismen zu aktivieren. Diese Selbstheilungskräfte sind Grundlage jeder Heilung. In diesem Zusammenhang nennt man heute häufig die Salutogenese (Kasten). Dabei geht es um die Frage, wie Gesundheit entsteht und wie Gesundheitsentstehung im konkreten Fall gefördert werden kann - als Alternative zum alleinigen Fokus auf der Entstehung von Krankheit (Pathogenese).

Gesundheit ist niemals ein statischer Zustand, sondern ein dynamischer Prozess. In der Humoralmedizin gilt, dass bei jeder chronischen Erkrankung der Einfluss der Melancholie (Schwarzgalle, Element Erde, kalt und trocken) mitzubehandeln ist. Die Chronizität einer Erkrankung geht mit Stagnation und einem Mangel an Vitalkräften einher. Chronische Erkrankungen brauchen Energie und wirken «zehrend» auf den Menschen. Diesem Aspekt muss in der Therapie Rechnung getragen werden. Alles, was

Tab. 1. Abhängiges und ganzheitliches Modell der Beziehung zwischen Patient/Patientin und medizinischen Fachpersonen [6]

\begin{tabular}{ll}
\hline Abhängiges Modell & Ganzheitliches Modell \\
\hline $\begin{array}{l}\text { Der Patient ist Laie. } \\
\text { Er ist unterlegen und unwissend. }\end{array}$ & $\begin{array}{l}\text { Der Patient ist als «Partner» anerkannt. } \\
\text { Er ist ebenbürtig und selbstständig. }\end{array}$ \\
\hline Uninformiert und unwissend & sachkundig werden, Fragen stellen \\
Unterwürfig & stark und integer \\
Passiver Zuschauer & Teilnehmer und Handelnder \\
Gibt Verantwortung ab & verantwortungsbewusst \\
Angepasst, abwartend & vorausdenkend \\
Beschwichtigt & bleibt handlungs- und entscheidungsfähig \\
Leicht führbar & behält die Führung des eigenen Lebens \\
Verletzlich, verletzbar & schafft Selbstvertrauen \\
Hilflos, Hilfe suchend & selbstständig, mutig \\
Wankelmütig, fragt nach Meinung anderer & entschlossen, sich selbst zu helfen \\
Ängstlich, unsicher & zuversichtlich und klar im Handeln \\
Resigniert & zufrieden, konstruktive Lebenseinstellung \\
\hline
\end{tabular}

Abb. 1. Bei chronischen Erkrankungen wird der Mensch mit seiner Sterblichkeit konfrontiert.

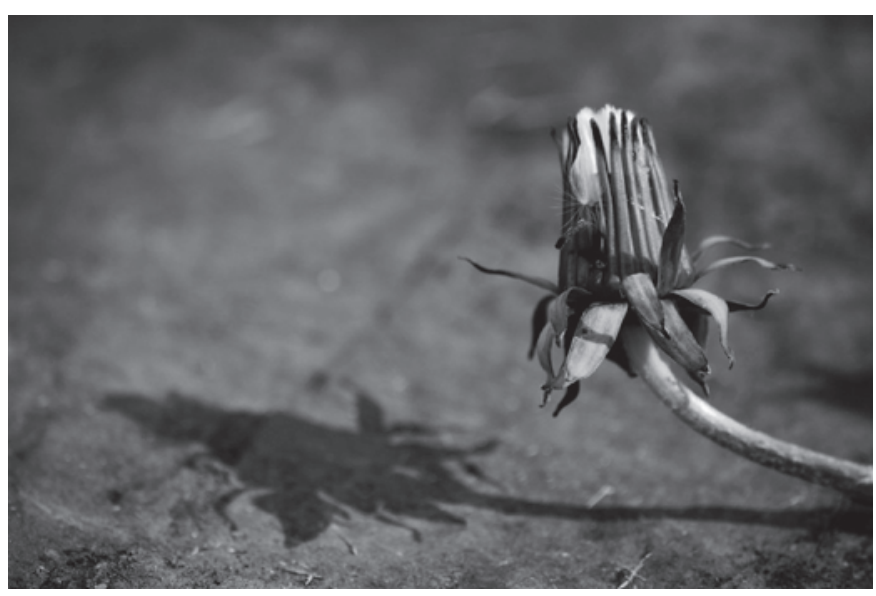

die Vitalität und die Lebensfreude fördert, soll in die Therapie von chronisch kranken Menschen miteinbezogen werden. Von Thomas von Aquin ist in diesem Zusammenhang überliefert: «Gesundheit ist weniger ein $\mathrm{Zu}$ stand als eine Haltung. Und sie gedeiht mit der Freude am Leben.»

\section{Konkrete Therapiemassnahmen}

- Förderung des psychischen Gleichgewichts, Ausgleich von Stimmungsschwankungen, genug Schlaf und Regeneration, Abbau von Stress (Störung der Immunfunktion durch die Cortison-Stimulation bei Stress), Entspannungsmassnahmen wie Yoga, Biofeedback, autogenes Training, Atemtherapie und viele andere.
- Magen-Darm-Trakt/Stoffwechsel: Essenziell ist die gastrointestinale Regulation, insbesondere in Bezug auf die Darmflora und das darmassoziierte Immunsystem (GALT). In der Traditionellen Europäischen Naturheilkunde (TEN) gilt der Magen als Initiator der Stoffwechselprozesse (Kochungen). Das Abdomen wird deshalb auch Quelle aller Quellen genannt. In der Traditionellen Chinesischen Medizin (TCM) steht der mittlere Erwärmer im Fokus.

- Unterstützung der Leber, die eine essenzielle Rolle bei der Reinigung und Elimination von Stoffwechselendprodukten, Medikamenten, Genussmitteln, Toxinen und anderen unphysiologischen Substanzen hat: Leberpflanzen wie Mariendis- 
Abb. 2. Getrocknete Blätter der Gundelrebe (Glechoma hederacea).

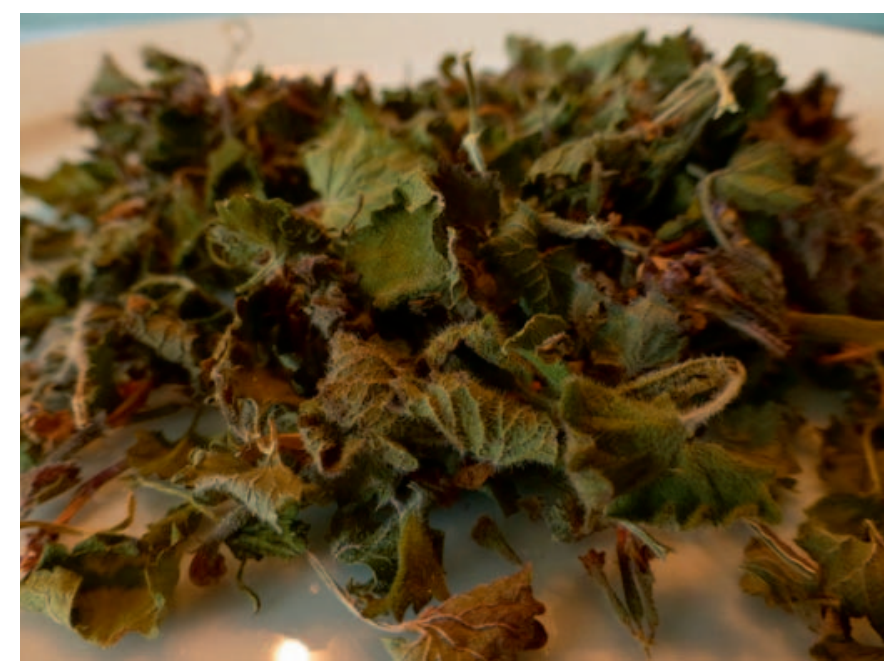

tel (Silybum marianum/Carduus marianus (Silymarin)) usw.

- Unterstützung der Nierenfunktion: Die Nieren gelten in der TCM als Sitz der Lebenskraft und in der TEN als Grundkraft und Initialfunken für das gesamte Stoffwechselgeschehen.

- Langfristig ausgewogene Versorgung mit Mikronährstoffen (Tab. 2).

- Reduktion der Glykosylierung von Proteinen mittels Reduktion der raffinierten Kohlenhydrate in der täglichen Ernährung, Verbesserung der Glukosetoleranz mit Zink, Chrom, Mangan, B-Vitaminen usw. sowie der Gabe von Carnosin (Dipeptid aus Beta-Alanin und L-Histidin), das durch die antioxidative, antiglykosilierende, aldehydneutralisierende und metallchelierende Wirkung vor Proteinmodifikationen schützt $[1,3]$.

- Ausgleich des Säure-Basen-Haushalts.

- Multimedikation: Die Tatsache, dass einerseits bei chronischen Erkrankungen oft viele Medikamente eingenommen werden, muss bei einer ganzheitlichen Therapie zwingend unter die Lupe genommen werden, denn das häufig beobachtete Phänomen der Multimedikation ist heute ein grosses Problem. Andererseits gilt es, die Alltagsqualität der Patienten zu erhalten. Beispiele naturheilkundlicher Alter- nativen zu chemischen Arzneimitteln sind Akupunktur, Schröpfen, Baunscheidtieren, Phytotherapie (Teufelskralle, Weihrauch, Weidenrinde), Moxa, Hydrotherapie, Chiropraktik, Fasten, Homöopathie, Spagyrik usw.

- Pankreasenzyme, die Immunkomplexe aufbrechen und damit chronische Entzündungen, Schmerzzustände und Gewebeschäden reduzieren.

- Hervorzuheben ist hier eine besondere Heilpflanze: Glechoma hederacea (Echt-Gundelrebe, Gundermann) (Abb. 2). Die Gundelrebe ist eine einheimische Heilpflanze aus der Familie der Lamiaceae (Lippenblütler) mit stoffwechselanregenden, antiphlogistischen und diuretischen Eigenschaften. Wie die meisten Vertreter aus der Familie der Lamiaceae wirkt die Gundelrebe auf die Wärmeregulation des menschlichen Organismus ein. Die Pflanze mit ihren lila-blauvioletten Blüten und den herz- bis nierenförmigen Blättern wächst an Wegund Waldrändern. Die Gundelrebe ist sowohl vermeintliches Unkraut als auch erdig-krautiges Küchengewürz. Sie gehört zu den typischen Frühlingspflanzen (traditionellerweise auch eines der neun Kräuter der Gründonnerstagssuppe «Grüne Neune»). Als Inhaltsstoffe sind ätherische Öle, Gerb- und Bitter-
Tab. 2. Die wichtigsten Komponenten einer langfristig ausgewogenen Versorgung mit Mikronährstoffen bei chronischen Erkrankungen [2,3]

Vitamin A, E und C, Vitamin B-Komplex

Carotinoide (Beta-Carotin, Lycopin)

Anthocyane

Coenzym Q10

Selen, Zink, Mangan

Magnesium

Omega-3-Fettsäuren

Phenole und phenolische Säuren

Flavonoide und Isoflavone

Catechine

Salicylate (Silber-Weide/Salix alba; Echtes

Mädesüss/Filipendula ulmaria)

Reservatrol (aus der Schale der roten

Weintrauben)

Quercetin (ebenfalls aus der roten Weintraube)

Curcuma (Gelbwurz/Curcuma longa)

Oligomere Proanthocyanidine (OPC) aus den

Schalen und den Kernen der roten Weintraube Epigallocatechin-Gallat (EGCG) aus grünem Tee Alpha-Liponsäure

Boswelliasäure (Weihrauch/Boswellia serrata)

Melatonin

Sterole und Steroline

Dehydroepiandrosteron (DHEA)

stoffe bekannt [6]. Neben ihrer Anwendung bei Erkrankungen der Atmungsorgane wie Schnupfen, Bronchitis, Nieren-Blasen-Beschwerden, Reizblase, bei Frühjahrskuren sowie bei Stoffwechselerkrankungen gilt sie als eine der grossen Heilerinnen bei chronischen Erkrankungen, wenn bei langwierigen, hartnäckigen und zehrenden Krankheitszuständen das Vertrauen in die eigene Lebendigkeit und die inneren Selbstheilungskräfte versiegt ist [7].

Das Wesen der kleinen und unscheinbaren Gundelrebe wird mit «Loslassen und Erneuerung, Gelassenheit und lebenserweckende Wärme» [7] beschrieben. Eine grosse Lebendigkeit und Wärme, die erstarrte Prozesse in ruhiger und gelassener Weise wieder in Gang zu bringen vermag, liegt in der Pflanze verborgen.

In der TEN wird die Gundelrebe als erwärmend, trocknend, reinigend und eröffnend beschrieben und gilt als warm und trocken im 2. Grad [8]. 


\section{Literatur}

1 Slazenger S: Inflammation, die stille Epidemie unserer Zeit. Naturheilpraxis 8/2013.

2 Burgerstein U-P, Schurgast H, Zimmermann M: Handbuch Nährstoffe. Stuttgart, Trias, 2012.

3 Nestroj I: Praxis der orthomolekularen Medizin. Stuttgart, Hippokrates, 2000.
4 Karl J: Neue Therapiekonzepte für die Praxis der Naturheilkunde. München, Pflaum, 1995.

5 Schünemann M: Umgang mit Schmerzmitteln. Naturheilpraxis 9/2014.

6 Raimann C, Ganz C, Garvelmann F, BertschiStahl HD, Fehr-Streule R: Grundlagen der Traditionellen Europäischen Naturheilkunde. Schiedlberg, Bacopa, 2012.
7 Kalbermatten R: Wesen und Signatur der Heilpflanzen. Aarau, AT, 2002.

8 Garvelmann F: Pflanzenheilkunde in der $\mathrm{Hu}$ moralmedizin. München, Pflaum, 2000. 